\title{
An Intervention Effect Analysis of Urban Transformation for Manhattan by Special Purpose District Enforcement
}

\author{
Jinho Park ${ }^{1}$, Hyoun-Joo Kim ${ }^{2}$ and Woo-Hyoung Lee ${ }^{3}$ \\ ${ }^{1}$ Global School of Media, Soongsil University, Seoul, 156-743, South Korea \\ ${ }^{2} J u n g-A n$ Architects., Sangdong, Suseong, Daegu, South Korea \\ ${ }^{3}$ Department of Architecture, Namseoul University, Cheonan, 331-707, \\ South Korea \\ 3wlee@nsu.ac.kr
}

\begin{abstract}
This study embodies the impact of institutional intervention of urban remodeling to study the quantitative correlation between urban planning and urban change. We also take a new perspective to differentiate our research from other theoretical studies by using urban data as a possibility resource that allows new interpretation of urban phenomena. In this study, a regional urban planning program for a specific purpose was implemented using the number of new constructions and renovations representing the physical urban transformation of the four boroughs in Manhattan, New York, and the index before and after a certain period Compare the pattern of change of. This will quantify the impact of urban change and provide an analytical base for implementing urban planning from a mathematical point of view.
\end{abstract}

Keywords: intervention time series analysis, ARIMA, exponential smoothing

\section{Introduction}

Urban transformation arises from the complex correlation between autonomous evolution and intentional governmental institutional interventions. The effectiveness of institutional interventions is unpredictable to date, since it is inadequate to quantify how to allow the interpretation of urban transformation. But with the remarkable growth of the digital industry these days, a huge amount of data is generated and collected at every moment, presenting various perspectives on the unique characteristics of urban phenomena. Therefore, data can be used as a material for mathematical analysis and more concrete and practical interpretation is possible.

A new research methodology based on an empirical analysis of urban operating mechanisms allows the understanding of the causes and effects of urban transformation patterns. [1]. It is a matter of course that there is a difference in the degree to which administrative intervention of the government-led system influences the statistical indicators of the city, for example, population, number of buildings, and real estate prices. However, there are complicated aspects to demonstrate how much it affects the indicators of urban change and how quantitatively it is. This is because it is unreasonable to simply state that an administrative system has influenced certain urban indicators, because urban change is a factor of change and there are many factors that affect each other.

Nonetheless, interpreting administrative intervention and urban change within a mathematical model has the following advantages. First, it can predict the impact of the policy when drafting the policy, which is helpful when drafting the policy plan and contents. In this study, we conducted a study on the system of the special purpose district of Manhattan, for example, as a tool to simulate the effects of the same system when applied

Received (October 7, 2017), Review Result (January 10, 2018), Accepted (January 30, 2018) 
to other cities. This can contribute to the successful settlement of the system, budget reduction, and social stability. Second, this study makes it possible to analyze the effect of the system on urban change by element. The analysis of other areas where the same system is applied can be analyzed to see how the system has affected the city, and this opens up a new horizon of urban studies.

\section{Our Approach}

The time series are a series of data points indexed in chronological order. Most generally, the time series is a sequence performed at equidistant points continuous in time. Thus it is a sequence of discrete-time data. Since the temporal change of building uses in Manhattan districts must be a type of time series, we adopt the time series analysis techniques to observe the characteristics of urban data such as temporal trend and event affect.

Interventions Time series analysis is a standard statistical method for evaluating the impact of interventions (usually planned policy changes) in time series of relevant outcome indicators. In interventional time series analysis, intervention is described as an event applied to a temporal phenomenon at a specific time. The intervention can be one and only one event such as 911 attacks or persistent event such as special purpose district program as in our urban transition data.

The ARIMA(Auto-regressive Integrated Moving Average) model is a kind of time series analysis technique, which is a generalization of the ARMA model which explains the current time series value using past observations and errors. This means that while the ARMA model is only applicable to the stationary series, it can be applied even if the analyzer features a slightly nonstationary series. A stable time series is a time series with the following three characteristics.

- The average is constant regardless of the trend of time

- Variance is invariable regardless of the time trend

- Covariance between two time points is independent of baseline

Whether or not a time series is stable is important because a time series must be a stable time series in a general time series analysis. If the time series is not stable, it should be analyzed after stabilizing the time series by using log or difference.

The non-seasonal ARIMA model is generally expressed as ARIMA (p, d, q), the parameters $p, d$ and $q$ are nonnegative integers, $p$ is the order of the autoregressive model. And $\mathrm{q}$ is the order of the moving average model. Seasonal ARIMA models are usually expressed as ARIMA (p, d, q) (P, D, Q) m, where $\mathrm{m}$ is the number of periods in each season and $\mathrm{P}, \mathrm{D}$ and $\mathrm{Q}$ are in capital letters. The ARIMA model uses a moving average. If two of the three terms are zero, you can refer to the model by deleting "AR", "I", or "MA" from the abbreviation describing the model based on nonzero parameters. For example, ARIMA $(1,0,0)$ is AR $(1)$, ARIMA $(0,1,0)$ is I $(1)$, and ARIMA $(0,0,1)$ is MA (1) [2].

A time series $\left\{Y_{t}\right\}$ can be given by

$$
Y_{t}=m_{t}+N_{t}
$$

, where $m_{t}$ is the change in the mean function and $Y_{t}$ is modeled as some ARIMA process which is underlying time series without any intervention. Intervention $m_{t}$ has very important role in analyzing the intervention effects. There are two kinds of events. The first is represented by the pulse function as a single event, like the 911 attack, and the second by the step function, which continues for a single time after it has occurred. 


$$
\begin{gathered}
S_{t}^{(T)}= \begin{cases}1 & , t \geq T \\
0 & \text { else }\end{cases} \\
P_{t}^{(T)}=S_{t}^{(T)}-S_{t-1}^{(T)}
\end{gathered}
$$

And the modulated change in mean function can formulated as

$m_{t}=\omega S_{t}^{(T)}$

, where $\omega$ is the unknown parameter change in the mean due to the invention.

We model the intervention effects for urban transformation by institutional intervention in pulse framework

$$
m_{t}=\omega_{0} P_{t}^{(T)}+\frac{\omega_{1}}{1-\omega_{2} B} P_{t}^{(T)}
$$

, where operator $B$ is the backshift operator and $B m_{t}=m_{t-1}$. This implies that the change in the mean takes place after a delay of one time unit and the effect dies out gradually. The first term of Equation (5) means the initial impact which happens in the beginning and the second term represents gradual accumulation of the impacts. $\omega_{2}$ is crucial parameter which represents consistency of impact delivery. The consistency can be formulated as

$$
m_{t}=\frac{\omega_{1}}{1-\omega_{2} B} P_{t}^{(T)} \Leftrightarrow m_{t}=\omega_{2} m_{t-1}+\omega_{1} P_{t}^{(T)}
$$

Equation (6) shows that parameter $\omega_{2}$ tells how much of the previous step affects the next step when moving from the previous step to the next step. In addition, parameter $\omega_{0}$ indicates how much impact was given at the beginning, and parameter $\omega_{1}$ indicates how much impact is added per step. All of these parameters are calculated as unknown by ARIMA analysis and are a key element for expressing intervention effects.

\section{Experimental Results}

The scope of this analysis covers new buildings and alterations in 4 districts, Chelsea, Clinton, Garment Center and Hudson Yards in Manhattan, New York from 1950 to 2015. To be greatly influenced by the appointment of a special purpose district as a city transition indicator obtained from PLUTO [3]. The district was appointed special purpose districts in 2005. As an analytical scheme, Holt-Winters method for exponential smoothing and ARIMA for intervention time series analysis are used. Use the time series analysis library implemented by R (Refer to Appendix). By applying various smoothing operators such as simple exponential smoothing and moving average, it is possible to observe time series trend of time series $[4,5]$. Exponential smoothing is commonly applied to smooth data because many window functions are in signal processing and act as low pass filters to remove high frequency noise [6].

In this paper, we discuss a method to quantify the socioeconomic transition of urban indices by intervention. Furthermore, the challenge is to open up a new problem scope to investigate the possibility of changing domains into the analysis space. The pre-intervention series (up to 2005 in this example) is modeled by ARIMA $(1,0,0)$ because the original data is assumed to be stationary. The applied change in mean function is a mixed form of one shot event and accumulated affects shown in Equation (5). As in Appendix, the parameter finding is performed by arimax function in TSA library of R. The function extracts the main parameters $\omega_{i}, i=1,2,3$ of the change in mean function. Figures (1)-(8) show the plots of raw data and the intervention effects. 


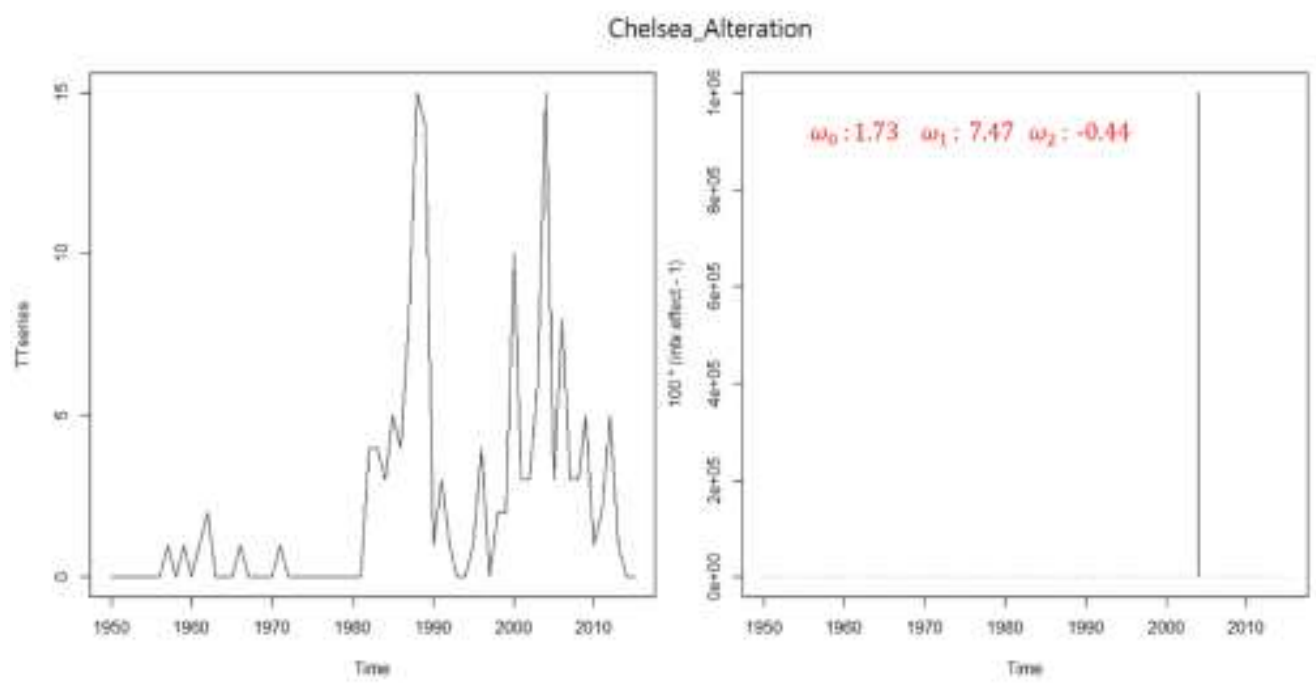

Figure 1. Alteration Data for Chelsea Borough of Manhattan, New York from 1950 to 2015 (Left) and Intervention Effects of SPD Enforced in 2005 (Right)

Figure 1 shows data on the number of reconstruction in the Chelsea area and analysis of the intervention effect. After a large increase in the late 1980s, the increase and decrease are repeated. Since the beginning of the SPD implementation period in 2005, the increase has been repeated, but the level is not so large. Since $\omega_{2}$ is -0.44 , it can be considered that there is almost no gradual increase. If $\omega_{2}$ is close to 1 , the result of the previous step is accumulated. This proves that the effect of the SPD is transient, but it can be seen that the width is quite large. $\omega_{0}$ of 1.73 indicates a significant intervention effect at the time of enforcement. $\omega_{1}$ is 7.47 means that the effect of the system is greater as time accumulates, but it is not meaningful because $\omega_{2}$ is negative.

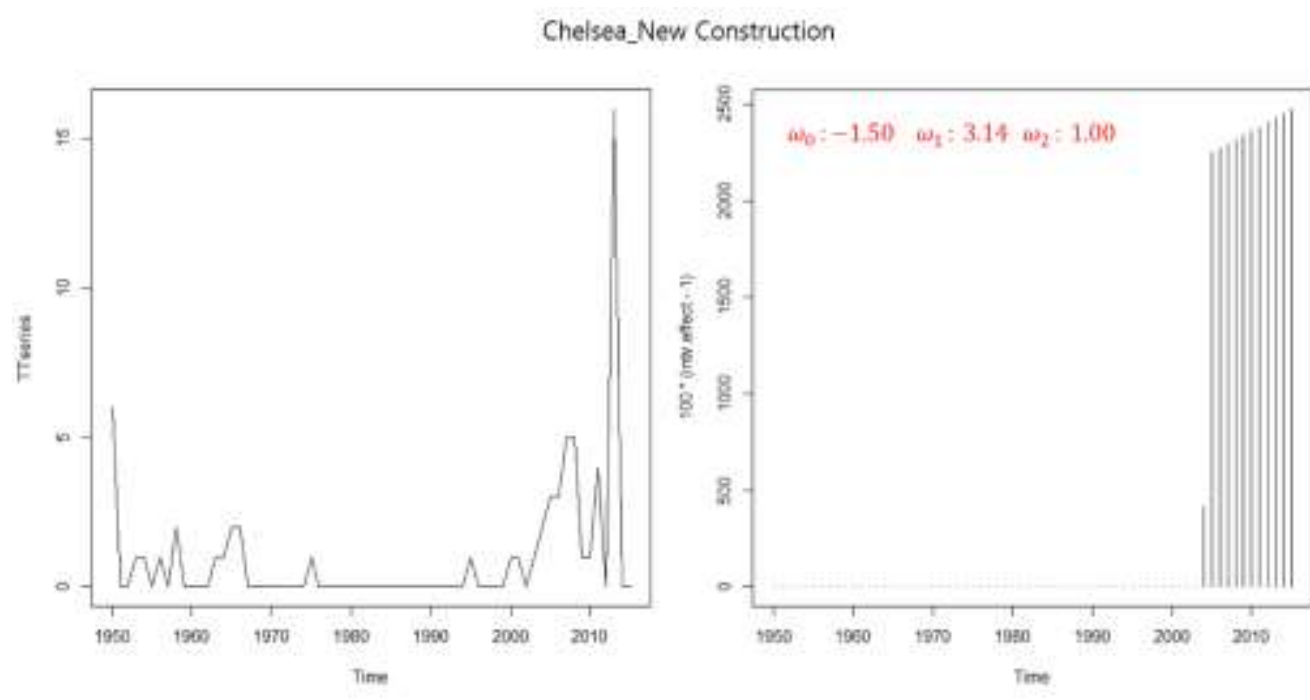

Figure 2. New Construction Data for Chelsea Borough from 1950 to 2015 (Left) and Intervention Effects of SPD enforced in 2005 (Right)

Figure 2 is a quantification of the effects of SPD on the number of new cases in the Chelsea region. It should be noted that raw data also shows that the number of new projects has increased from the 2005 SPD implementation. In the analysis result, it is found that $\omega_{2}$ is 1.00 indicating persistence, and thus it shows an ideal cumulative increase form. Since 
$\omega_{0}$ is negative, the increase is small in the first year of implementation, and the fact that $\omega_{1}$ is 3.14 means that the effect of implementation of the system increases with time. This result, among all the analysis results, is the ideal influence analysis in terms of sustainability.

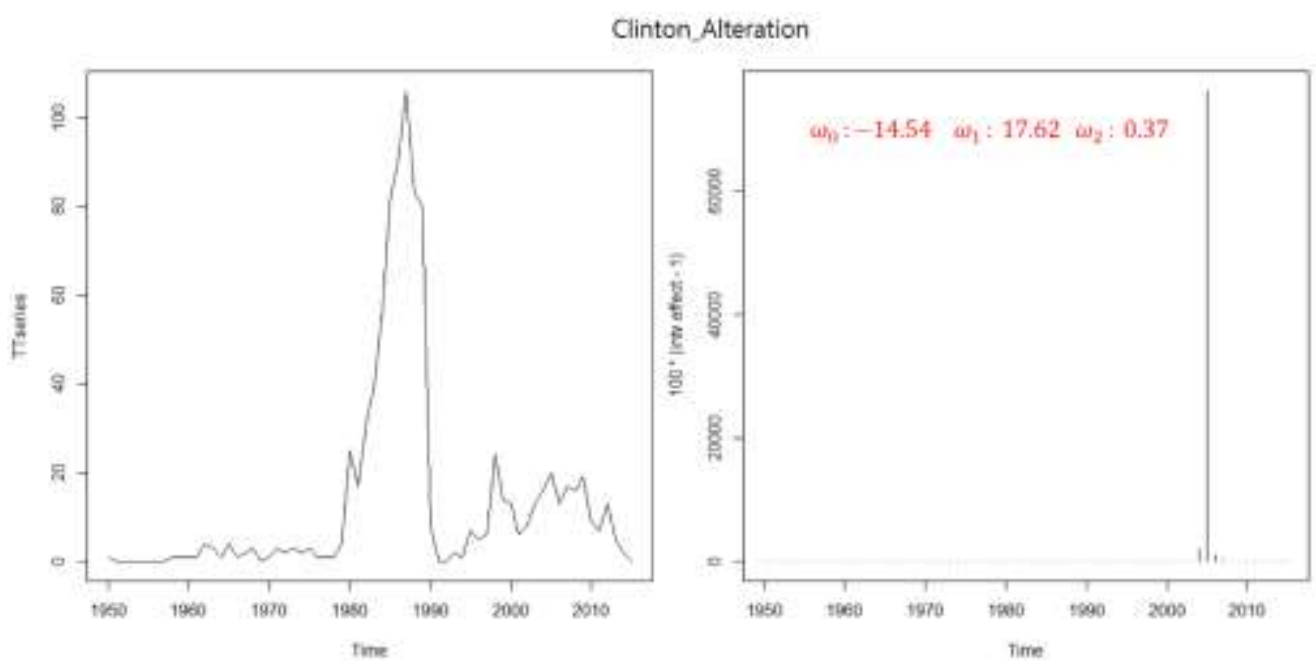

Figure 3. Alteration Data for Clinton Borough from 1950 to 2015 (Left) and Intervention Effects of SPD enforced in 2005 (Right)

Figure 3 shows the restructuring situation of the Clinton area and the corresponding intervention effect analysis. $\omega_{2}$ is 0.37 , so it has low persistence, negative effect at the time of implementation, and $\omega_{1}$ is very large, so it has great influence over time. It is analyzed as having a temporary effect, and it is hard to say that it was affected specially as seen in the raw data.

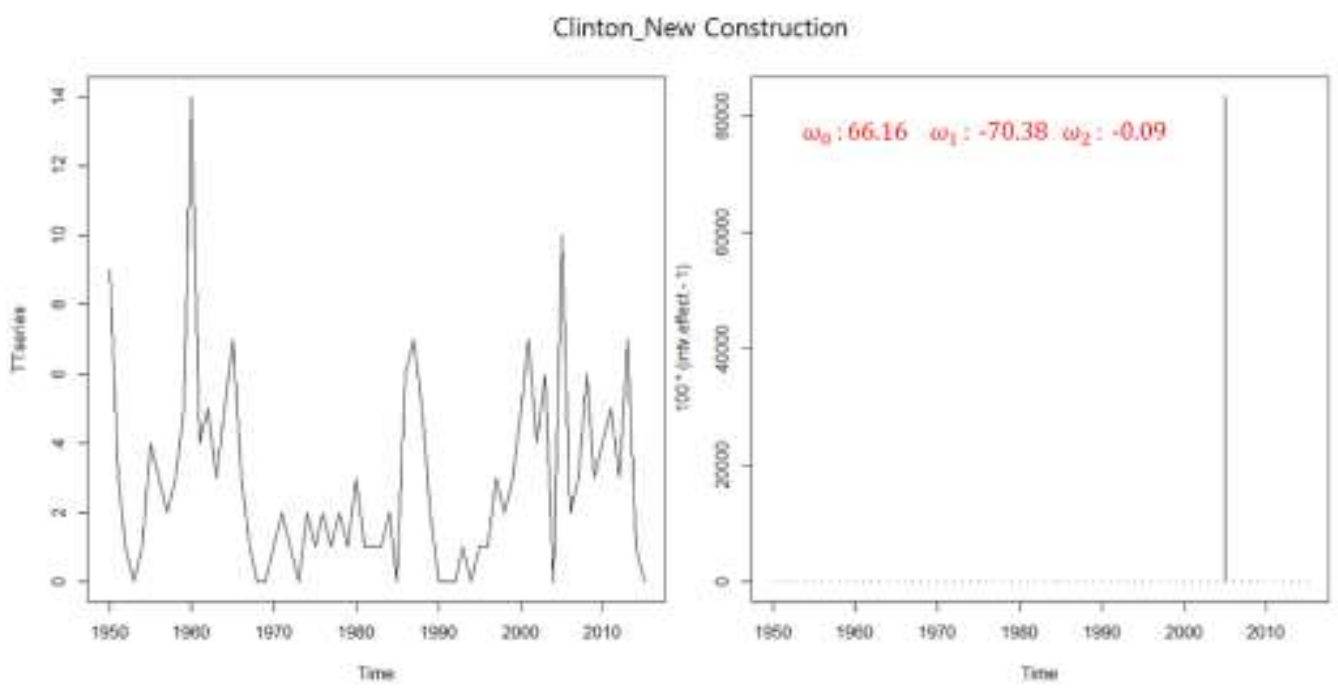

Figure 4. New Construction Data for Clinton Borough from 1950 to 2015 (Left) and Intervention Effects of SPD enforced in 2005 (Right)

For the Clinton area, there is no significant difference in the number of new constructions due to SPD (Figure 4). In particular, $\omega_{2}=-0.09$ means that there is almost no persistence, and in fact, the intervention effects graph has temporarily increased, but there is no indication that the effect is transmitted to the next step. $\omega_{0}$ is very large at 66.16 , but it can 
not be concluded that it was influential at the beginning of the experiment. The reasons may have been increased to other factors other than those caused by SPD in 2004 and the following year.

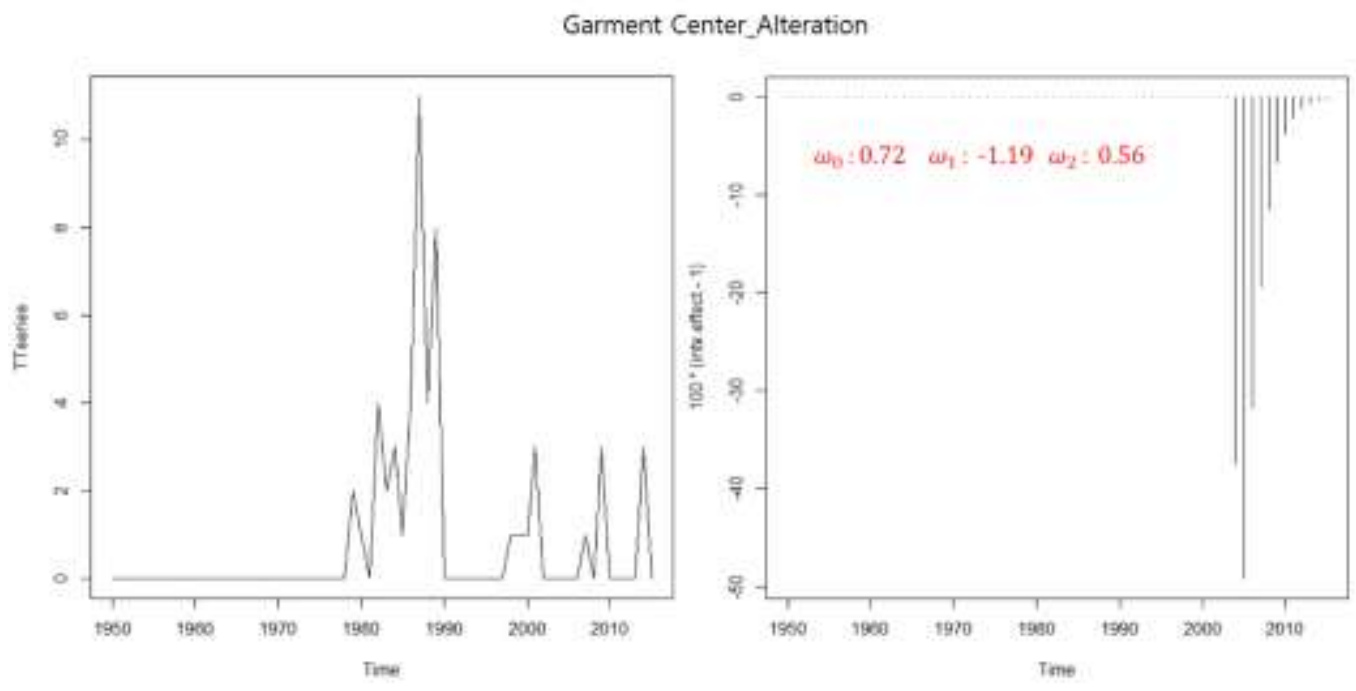

Figure 5. Alteration Data for Garment Center Borough from 1950 to 2015 (Left) and Intervention Effects of SPD enforced in 2005 (Right)

In the case of the reconstruction data of the Garment Center area, unlike the previous case, the unusual thing is that the influence is negative (Figure 5). This means that the intervention has had an adverse effect. In other words, if there were no intervention, the number of reconstructions could be increased, and the number of reconstruction was reduced due to the occurrence of SPD events. Since $\omega_{2}$ is 0.56 , interesting result is obtained in which the negative effect is transited according to time anyway.

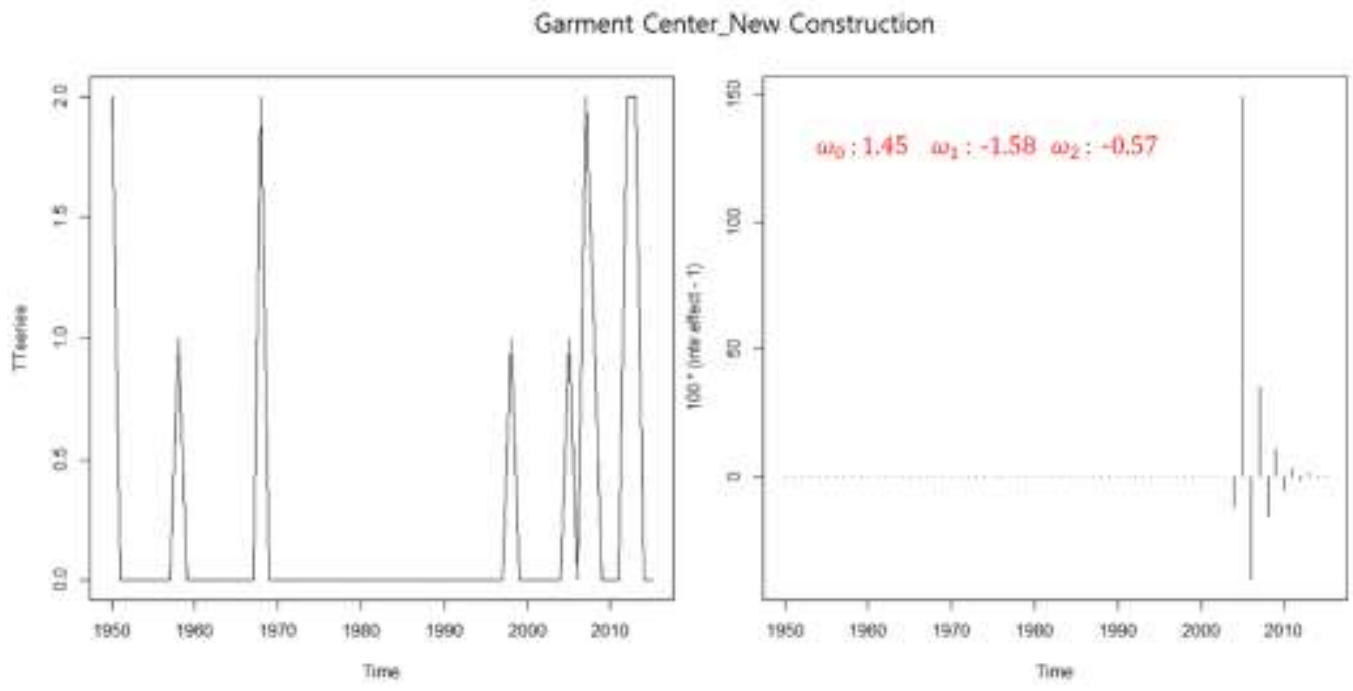

Figure 6. New Construction Data for Garment Center Borough from 1950 to 2015 (Left) and Intervention Effects of SPD enforced in 2005 (Right)

The new data from the Garment Center also show interesting results (Figure 6). Once in raw data, the number of new constructions is noticeably increasing since the application of SPD. In any case, the application of the SPD system has affected the number of new projects. 
However, it can be seen that the increase in the number of new constructions is not maintained, and the intermittence is greatly reduced. In other words, the effect is enormous, but on the contrary, it is greatly reduced. This also appears in intervention effects analysis (Right in Figure 6). As long as $\omega_{2}$ speaks minus, the increase and decrease go back and forth. $\omega_{1}=-1.58$ means that the influence decreases over time.

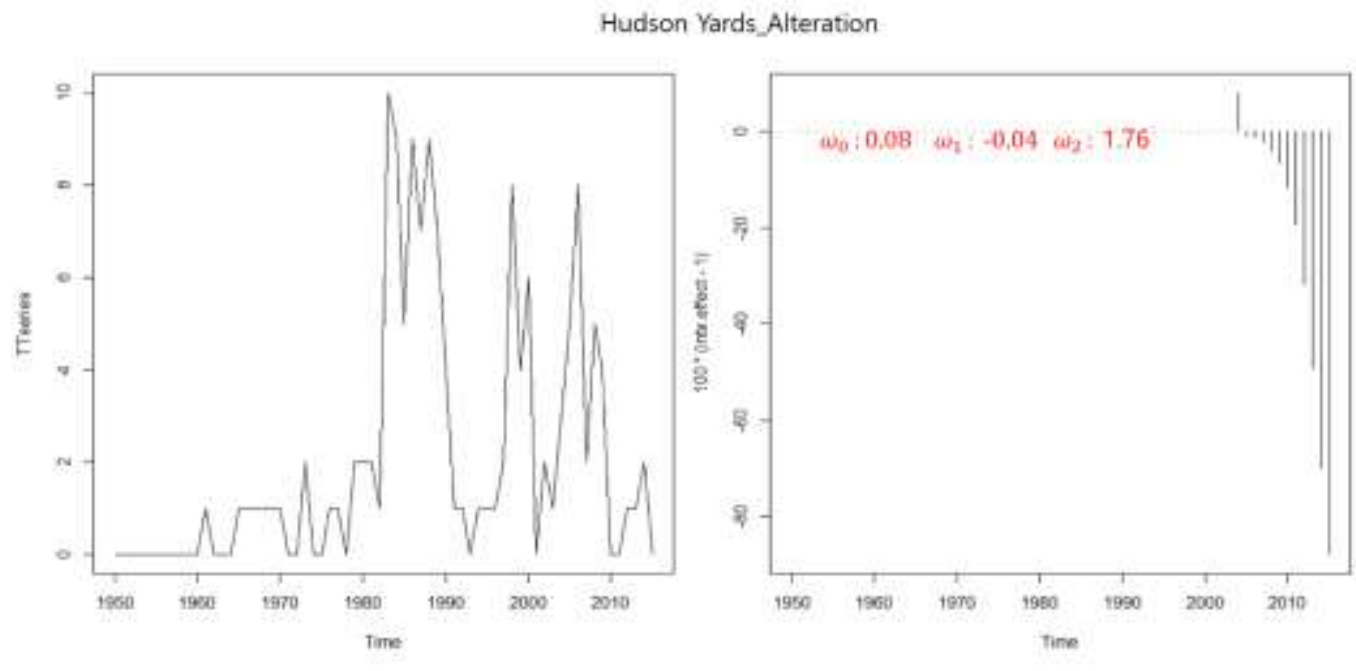

Figure 7. Alteration Data for Hudson Yards Borough from 1950 to 2015 (Left) and Intervention Effects of SPD enforced in 2005 (Right)

Figure 7 shows the reconstruction situation of Hudson Yards. $\omega_{1}=-0.04$ means that there is almost no effect given repeatedly over time. $\omega_{0}=0.08$ also means that there is little impact at the start. However, $\omega_{2}=1.76$ means that intervention in the system continues to grow over time. As in the raw graphs, as in the intervention effects graph, the number of reconstructions is decreasing. In other words, the SPD continues to have a negative impact on the reconstruction of the area, and the extent is also increasing.

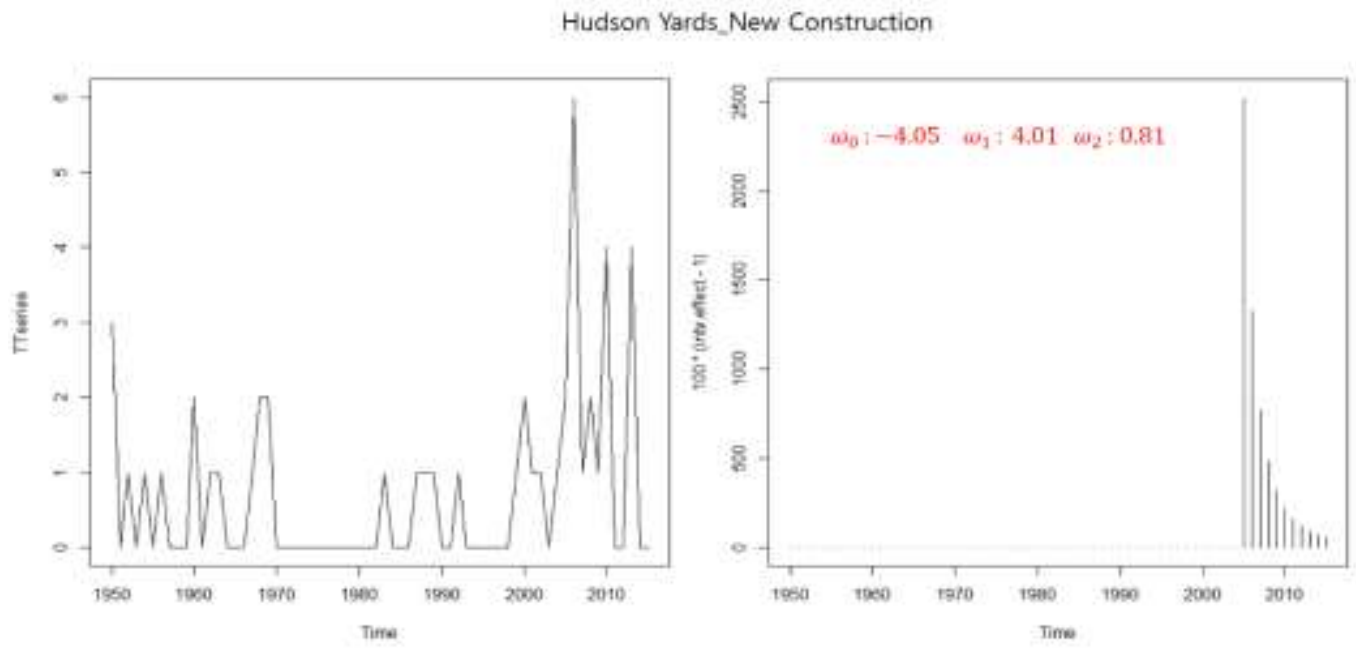

Figure 8. New Construction Data for Hudson Yards Borough from 1950 to 2015 (Left) and Intervention Effects of SPD enforced in 2005 (Right)

The number of Hudson Yards new constructions shows a case where the effect of SPD is reduced (Figure 8). The first year of implementation had a negative impact on new construction $\left(\omega_{0}=-4.05\right)$. However, thereafter, the overall number of new constructions 
increased significantly $\left(\omega_{1}=4.01\right)$, indicating that the effects were long lasting but accumulating with a slight decrease $\left(\omega_{2}=0.81\right)$.

\section{Table 1. [Alteration Case] Coefficients of the Change in Mean Function in Equation 5}

\begin{tabular}{|r|r|r|r|r|}
\hline & Chelsea & \multicolumn{1}{|c|}{ Clinton } & \multicolumn{1}{c|}{$\begin{array}{c}\text { Garmen } \\
\text { Center }\end{array}$} & \multicolumn{1}{c|}{$\begin{array}{c}\text { Hudson } \\
\text { Yards }\end{array}$} \\
\hline$\omega_{0}$ & 1.73 & -14.54 & 0.72 & 0.08 \\
\hline$\omega_{1}$ & 7.47 & 17.62 & -1.19 & -0.04 \\
\hline$\omega_{2}$ & -0.44 & 0.37 & 0.56 & 1.76 \\
\hline
\end{tabular}

The results of the experiment are summarized in Table 1. The initial intervention had a major impact on Clinton's negative direction, and Garment Center and Hudson Yards had little impact early on. In terms of sustainability, Hudson Yards showed meaningful results. Clinton and the Garment Center know that the impact of intervention is temporary because of the small persistence. In the case of Clinton, $\omega_{1}$ is 17.62 , which is considered to have a great influence on the step progress, but it is not meaningful analysis if $\omega_{0}$ is -14.54 .

\section{Table 2. [New Construction Case] Coefficients of the Change in Mean Function in Equation 5}

\begin{tabular}{|r|r|r|r|r|}
\hline & \multicolumn{1}{|c|}{ Chelsea } & \multicolumn{1}{|c|}{ Clinton } & \multicolumn{1}{c|}{$\begin{array}{c}\text { Garmen } \\
\text { Center }\end{array}$} & \multicolumn{1}{c|}{$\begin{array}{c}\text { Hudson } \\
\text { Yards }\end{array}$} \\
\hline$\omega_{0}$ & -1.50 & 66.16 & 1.45 & -4.05 \\
\hline$\omega_{1}$ & 3.14 & -70.38 & -1.58 & 4.01 \\
\hline$\omega_{2}$ & 1.00 & -0.09 & -0.57 & 0.81 \\
\hline
\end{tabular}

Chelsea region is the best of all cases in the persistence aspect $\left(\omega_{2}=1.00\right)$ (Table 2). The effect of institutional intervention was the longest and stable effect. On the other hand, Clinton's new construction can be judged to have little impact on institutional intervention, as it has resulted in excessive results similar to reconstruction. Hudson Yards also has some persistence $\left(\omega_{2}=0.81\right)$ but gradually decreases.

\section{Conclusion}

We conducted quantitative research in this study through statistical models as to how the application of the system would affect its application area. As an example, we have collected data for the last 50 years in four regions of Manhattan, and observed the Special Purpose Districts in 2005. Among the various city-related data, the number of new construction and reconstruction that can be said to be a measure of urban revitalization was considered as the target element. The ARIMA model, which is widely used in the analysis of time series data, was used to analyze how much the system intervention affected. Intervention effect at the initial start, persistence, etc., and analyzed the regional data. Our attempt is limited to regions and data types, so it can be extended to other cities and other urban factors. 


\section{Appendix: Our R-code for Intervention Effects Analysis}

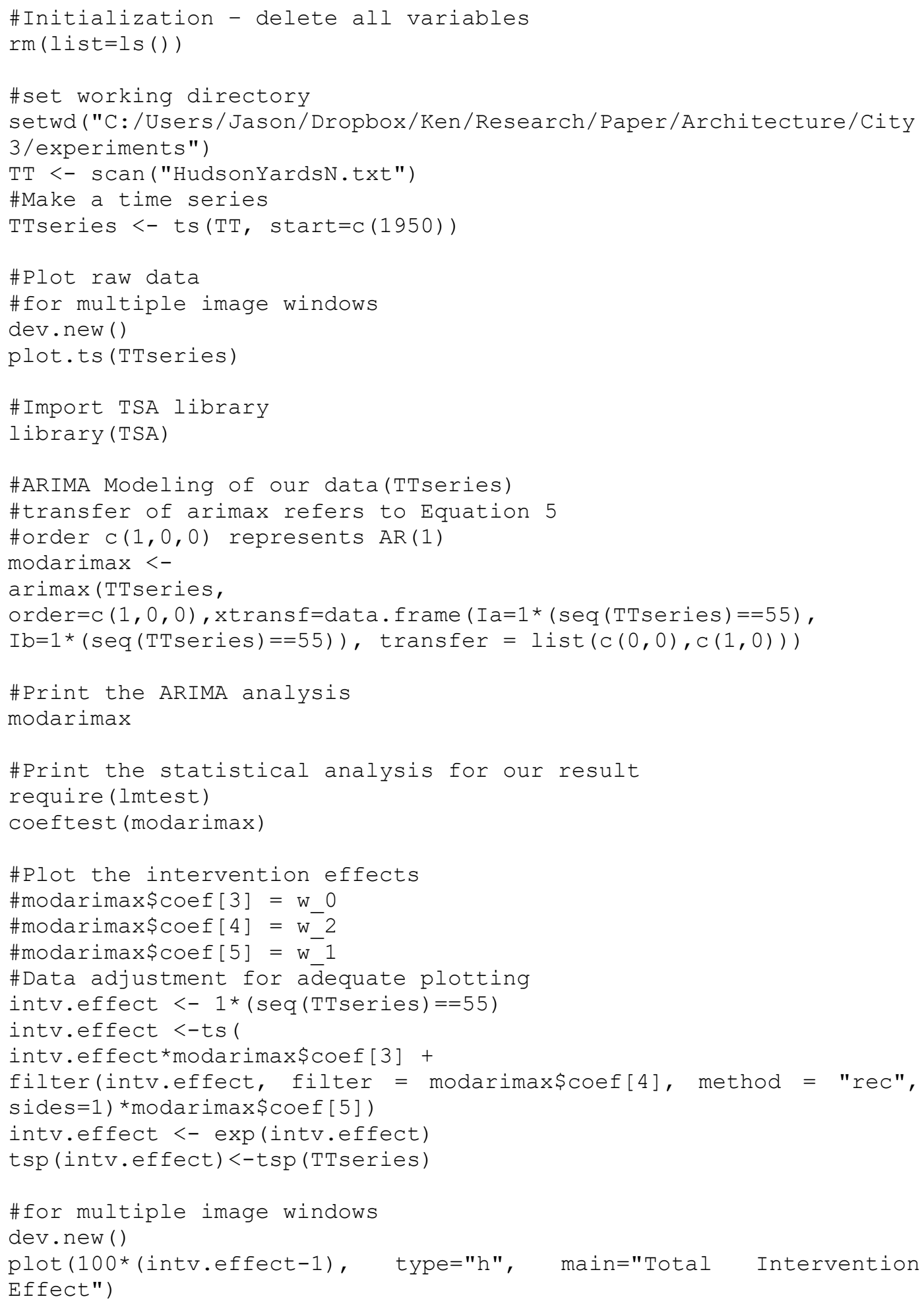

\section{Acknowledgements}

This research was supported by Basic Science Research Program through the National Research Foundation of Korea (NRF) Funded by Ministry of Education (2017R1D1A3B03034586). This paper is a revised and extended version of a paper entitled "A Mathematical Interpretation on Correlation between Institutional Intervention and 
Urban Transformation" presented at 9th International Mega-Conference on Future Generation Information Technology (FGIT 2017) which was held in Daejeon University, Korea, December 21 - 23, 2017.

\section{References}

[1] W. H. Lee, "Analysis of the Interrelation between Urban Transitional Patterns and Urban Programs", International Journal of Smart Home, vol. 10, no. 7, (2016), pp. 39-46.

[2] https://en.wikipedia.org/wiki/Autoregressive_integrated_moving_average

[3] http://www1.nyc.gov/site/planning/data-maps/open-data.page

[4] A. Pankratz, "Forecasting with Univariate Box-Jenkins models: Concepts and Cases", John Wiley \& Sons, vol. 224, (2009).

[5] D. C. Montgomery and L. A. Johnson, "Forecasting and Time Series Analysis", McGraw-Hill Companies, (1990).

[6] P. S. Kalekar, "Time Series Forecasting Using Holt-winters Exponential Smoothing”, Kanwal Rekhi School of Information Technology 4329008, (2004), pp.1-13.

[7] J. Park, H. J. Kim and W. H. Lee, "A Mathematical Interpretation on Correlation between Institutional Intervention and Urban Transformation”, Advanced Science and Technology Letters, vol.148(FGIT 2017), (2017), pp.7-10.

[8] "Diagnostic Ultrasound: Principles and Instruments", Journal of Nanosci. Nanotechnology., vol. 3, no. 2, (2003), pp. 75-80.

\section{Authors}

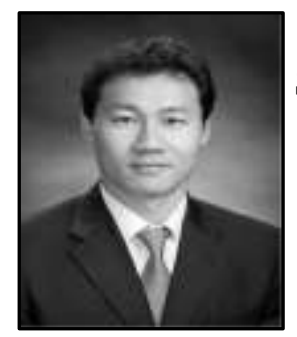

Jinho Park, Associate Professor at Soongsil University, Korea, South Korea

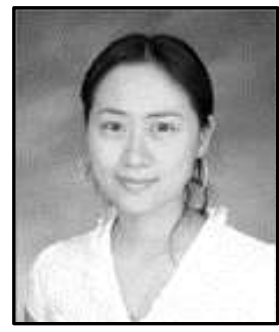

Hyounjoo Kim, Ph.D, Vice Director at JUNGAN Architects, Daegu, South Korea

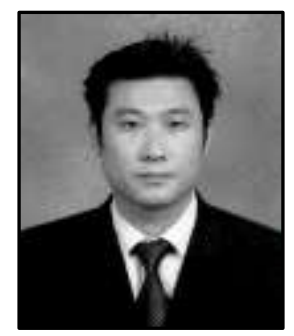

Woo-Hyoung Lee, Associate Professor at Namseoul University, Cheonan South Korea 